\title{
Are all wines made from various grape varieties beneficial in the prevention of myocardial infarction and stroke?
}

\author{
Masahiro Iwasaki ${ }^{1}$, Masahiro Murakami ${ }^{(D)}$, Yoshinobu ljiri $^{3}$, Muneshige Shimizu $^{4}$ \& \\ Junichiro Yamamoto*, 5 (iD \\ ${ }^{1}$ Division of Nutrition \& Metabolism, Original Nutrition Co., Ltd, Osaka 532-0002, Japan \\ ${ }^{2}$ Faculty of Pharmacy, Osaka Ohtani University, Osaka 584-8540, Japan \\ ${ }^{3}$ Department of Health and Nutrition, Osaka Shoin Women's University, Osaka 577-8550, Japan \\ ${ }^{4}$ Department of Fisheries, School of Marine Science and Technology, Tokai University, Shizuoka 424-8610, Japan \\ ${ }^{5}$ Kobe Gakuin University (Professor Emeritus), Kobe 651-2180, Japan \\ *Author for correspondence: yamamoto@nutr.kobegakuin.ac.jp
}

\begin{abstract}
Aim: Epidemiologic studies support the assumption (French paradox hypothesis) that drinking red wine is beneficial in the prevention of cardiovascular diseases. Our recent works however cast doubt on such claim. Earlier we have shown that the antithrombotic activity of various fruits and vegetables mainly depends on their varieties. For this reason, several varieties of red and white grapes were tested for antithrombotic effect in animal experiments. Results: Antithrombotic effect of 45 red and white grape varieties were assessed in the present study. Out of the 45 , one red grape variety showed antithrombotic effect, while the majority of red and white grape varieties enhanced thrombosis. Conclusion: Most red and white grape varieties enhanced thrombotic activity of blood.
\end{abstract}

Lay abstract: Red wine is widely believed to prevent heart attack and stroke as claimed by a French Paradox hypothesis. Antithrombotic effect of 45 grape varieties was measured in the present study. Only very few red and white varieties inhibited the experimentally induced thrombosis while the majority of the tested varieties enhanced thrombotic activity of blood. Thus, our findings challenge the prevailing claims of the French Paradox.

First draft submitted: 8 June 2020; Accepted for publication: 8 October 2020; Published online: 9 November 2020

Keywords: cardiovascular disease $\bullet$ fibrinolysis $\bullet$ grape $\bullet$ platelet aggregation $\bullet$ red wine $\bullet$ shear stress $\bullet$ stroke $\bullet$ thrombosis

Atherothrombosis plays important role in lifestyle-related myocardial infarction and stroke. Prevention of these diseases is an important social task in many countries. It has been shown that an appropriate diet may play an important role in the prevention of cardiovascular diseases. These studies have suggested that suitable intake of fruits and vegetable may prevent such diseases [1-5] and accordingly, many countries' health authorities have issued dietary guidelines [6-9]. Fruits and vegetables are classified into 'healthy' and 'less healthy' ones and they are recommended or advised against to their consumption accordingly [10].

Earlier we surveyed clinically useful blood tests for selecting fruits and vegetables for antithrombotic activity. Tests using anticoagulated blood have widely been used but they are not pathologically relevant and suitable for assessing thrombotic status of humans [11-13]. Blood tests using nonanticoagulated (native) blood and inducing thrombus formation solely by shear forces were developed in 1970 [14-21]. Recently, a commercially available point-of-care thrombosis test was shown to have pathological relevance to human arterial thrombosis [22-40].

Death from cardiovascular diseases in French people, who are taking thrombogenic high fat diet is surprisingly lower than in other European countries, although the latter take high-fat diet. Renaud and colleagues proposed that this may be due to the popular wine consumption of the French people (French paradox) [41]. Folts and colleagues demonstrated that red wine prepared from a special grape variety and the Welch's natural purple grape 
juice inhibited thrombus formation in animals (in vivo) and suggested that this may be due to some molecules such as polyphenols [42-45].

The aim of the present study was to test large number of grape varieties by a shear-induced thrombosis/thrombolysis test (Global Thrombosis Test) in order to assess the dependence of antithrombotic effect on the tested grape-varieties.

\section{Materials \& methods}

Animals

13-week-old male Wistar ST rats (Japan SLC Co. Ltd, Hamamatsu, Japan) and 10-week-old male C57BL/6 mice (Japan SLC Co. Ltd, Hamamatsu, Japan) were purchased 1 week before the scheduled experiments. Animals were maintained in compliance with the 'Guiding Principles for the Care and Use of Animals in the field of Physiological Sciences,' published by Physiological Society of Japan. The protocol was approved by the Animal Experiment Committee of Kobe Gakuin University.

\section{Grapes}

Eighteen grape varieties, (red) Asama merlot, Gamay noir, Cabernet sauvignon (Man), Cabernet franc, Syrah, Zenkoji, Pinot noir, Muscat bailey A (Man), Merlot, (white) Kerner, Shinano riesling, Chardonnay (Man), Sylvaner, Semillon, Sauvignon blanc, Pinot blanc, Muller thurgau, Riesling were donated from Manns wine Co., Ltd Japan; four varieties, (red) Cabernet sauvignon (Mer), Koshu, Muscat bailey A (Mer) and (white) Chardonnay (Mer) from Mercian Co. Ltd Yamanasi, Japan and twenty three varieties, (red) Aki queen, Oriental star, Kaiji, Charbono, Campbell early, Kyoho, Concord, Sunny rouge, Delaware, North red, Honey seedless, Honey black, Pione, Muscat bailey A (Nat), (white) Italia, Onsolo, Shine muscat, Dearing, Neo muscat, Honey venus, Pannonia kincse, Muscat of Alexandria, Megumi from National agriculture and food research organization.

\section{Preparation of grape juice filtrate}

Several clusters of grapes were collected from various portions of one tree and from several trees to avoid deviation. Grapes with skin were washed crushed in a mortar at room temperature. The juice was centrifuged at $3000 \mathrm{rpm}$ for $15 \mathrm{~min}$ at $4^{\circ} \mathrm{C}$. The supernatant was filtered (pore size $5 \mu \mathrm{m}$, FP 30/5.0 CN-S, BM Instrument Co. Ltd, Japan) and the clear filtrates were stored at $-80^{\circ} \mathrm{C}$ until use.

\section{In vitro assessment of platelet rich thrombotic \& endogenous thrombolytic activities by the Global Thrombosis Test}

The Global Thrombosis Test (GTT) (Thromboquest Ltd, London, UK) as described in detail earlier [46-49] was used to assess the antithrombotic effect of various grapes. The principle of this test had been described. Briefly, when nonanticoagulated blood flows through narrow channels, platelets are activated by high shear forces. Downstream from this point, the slow rate and turbulent flow favors the shear-activated platelets to aggregate and stabilized by the formed fibrin. Subsequently, the fibrin-stabilized platelet aggregates (thrombi) occlude the exit channels, causing the blood flow to be arrested. The instrument detects the time interval $(\mathrm{d}, \mathrm{s})$ between consecutive blood drops. At the start, blood flow is rapid and hence (d) is small. Subsequently, the flow rate decreases and hence (d) increases. When the actual (d) exceeds the default $15 \mathrm{~s}$, the instrument displays 'Occlusion Time (OT, s).' Subsequently, the flow is restored due to thrombolysis, and is indicated by the detection of the first blood drop as 'Lysis Time (LT, s).' In the present measurements, blood was withdrawn from the abdominal aorta of rats 30 min after Nembutal anesthesia (pentobarbital sodium, $60 \mathrm{mg} / \mathrm{kg}$, i.m.) and diluted with saline in 1:1 ratio. $0.4 \mathrm{ml}$ grape filtrate or $0.4 \mathrm{ml}$ saline (control) were added to $3.6 \mathrm{ml}$ of the two-times saline-diluted blood, mixed and transferred to the GTT test tube within $15 \mathrm{~s}$ following the withdrawal of blood. Four tubes containing various concentrations of grape filtrates and one control were assessed simultaneously in the four-channel instrument and the tests were repeated six times for each sample $(n=6)$. Prolonged OT suggests inhibition while shortened OT indicates enhanced thrombus formation, respectively. Prolonged LT suggests inhibited while shortened LT indicates enhanced thrombolysis, respectively. In case that the filtrate added to blood prolongs OT and shortens LT compared with saline, the result was thought to predict that filtrate has antithrombotic activity. In case that the filtrate prolongs OT but gives no effect on LT, the result was thought to predict that filtrate has antithrombotic activity. In case that the filtrate prolongs OT and prolongs LT, the result was thought not to predict the activity. This has to be determined by the in vivo test. In case that the filtrate shortens OT and prolongs LT, the result was 
thought to predict that the filtrate has prothrombotic activity. In case that the filtrate shortens OT but gives no effect on LT, the result was thought to predict that the filtrate has prothrombotic activity. In case that the filtrate shortens OT and shortens LT, the result was thought not to predict the activity. This has to be determined by the in vivo test. Thus, overall antithrombotic/prothrombotic activities are determined by the balance between the effects on thrombus formation and lysis. Finally, this is confirmed by the employed in vivo test, as described below.

\section{In vivo assessment of arterial thrombotic activity \& endogenous thrombolytic activity by Helium-Neon laser-induced thrombosis in mice}

The Helium-Neon laser-induced thrombosis method has been described in detail [46-50]. In brief, the left femoral artery of an anaesthetized mouse was exposed, and Evans blue dye was injected intravenously. The center of the exposed carotid artery was irradiated with the laser beam, and formation of thrombus at the site of irradiation was monitored and recorded on videotape. Images of the outlined thrombus were computer-analyzed at intervals of 10 s. The area of thrombus was delineated, and the thrombus mass was calculated by multiplying gray scale and area using Image J software (Image Processing and Analysis Java version 1.30, National Institutes of Health, Maryland, USA). Thrombotic status was expressed as the total sum of thrombus mass after the first 10 min of irradiation.

\section{In vivo assessment of antithrombotic activity of grape filtrates after oral administration}

Procedure has been described earlier in detail. In brief, filtrates and controls (distilled water) $(3.85 \mathrm{ml} / \mathrm{kg}$ body weight) were orally administered to animals through a gastric tube. A second dose using the same volume of filtrate or water $(3.85 \mathrm{ml} / \mathrm{kg}$ body weight $)$ was given $30 \mathrm{~min}$ later. Mice were anaesthetized and the laser-induced thrombosis experiments commenced $90 \mathrm{~min}$ after the second oral treatment. The antithrombotic or prothrombotic effects were assessed by measuring the volume of the thrombus mass. Compared with controls, reduced volume of thrombus mass indicated antithrombotic while increased volume suggested prothrombotic effect.

\section{Measurement of antioxidant activity}

Antioxidant activity was measured by chemiluminometry (Luminometer AB-2200; ATTO Co. Ltd, Tokyo, Japan). The reaction mixtures were (A) positive control, (B) negative control and (C) sample mixture. For (A), $150 \mu \mathrm{l}$ $100 \mathrm{mmol} / 1$ phosphate buffer and $60 \mu \mathrm{l}$ xanthine oxidase solution (XOD; $0.1 \mathrm{~m} / \mathrm{ml}$; Sigma-Aldrich Co, Ltd, MO, USA) were kept at $37^{\circ} \mathrm{C}$ for $1 \mathrm{~min}$. Ten microliters of 2-methyl-6-p-methoxyphenylethynylimidazopyrazione (MPEC) (ATTO Co, Ltd), $30 \mu \mathrm{l}$ saline and $50 \mu \mathrm{l} 3.6 \mathrm{mmol} / \mathrm{l}$ hypoxanthine were added and mixed for $1 \mathrm{~min}$. Measurements were performed over $45 \mathrm{~s}$ at $37^{\circ} \mathrm{C}$. For (B), XOD and saline were replaced with phosphate buffer and the test sample, respectively. For (C), $10 \mu \mathrm{l}$ saline in (A) was replaced with the test sample. Antioxidant activity was expressed as units of superoxide dismutase (SOD, units/ml filtrate) using a standard curve. Five parallel measurements were obtained from each sample.

\section{Measurement of polyphenol content in grape varieties}

Control and test sample mixtures were prepared in a similar manner to those described for the measurement of antioxidant activity. Chlorogenic acid (Sigma-Aldrich Co, Ltd) was used as the standard polyphenolic acid. A mixture consisting of $3.2 \mathrm{ml}$ distilled water, $20 \mu \mathrm{l}$ filtrate sample or the standard polyphenolic solutions, $200 \mu \mathrm{l}$ Folin-Dennis reagent and $400 \mu \mathrm{l}$ saturated $\mathrm{Na}_{2} \mathrm{CO}_{3}$ solution was kept at room temperature for $30 \mathrm{~min}$ and changes in absorbance at $760 \mathrm{~nm}$ were read using a spectrophotometer (Ubest-35; Jasco Co., Tokyo, Japan). The polyphenolic content was expressed as chlorogenic acid concentration. Triplicate measurements were obtained from each test sample.

\section{Statistical analysis}

Analysis was performed by a statistical package software (Unistat Light 5.6, Unistat Ltd, London, UK). Occlusion time (OT: raw data) and lysis time (LT; logarithmic data) were analyzed by repeated ANOVA, followed by post hoc, Dunnett. Thrombus mass in the laser-induced experiments was analyzed by ANOVA, followed by post hoc, Dunnett. Correlations of polyphenol contents/antioxidant activity and OT/LT were analyzed by Pearson Correlation Test. Results were expressed as means \pm SEM. $\mathrm{p}<0.05$ was considered as statistically significant. 

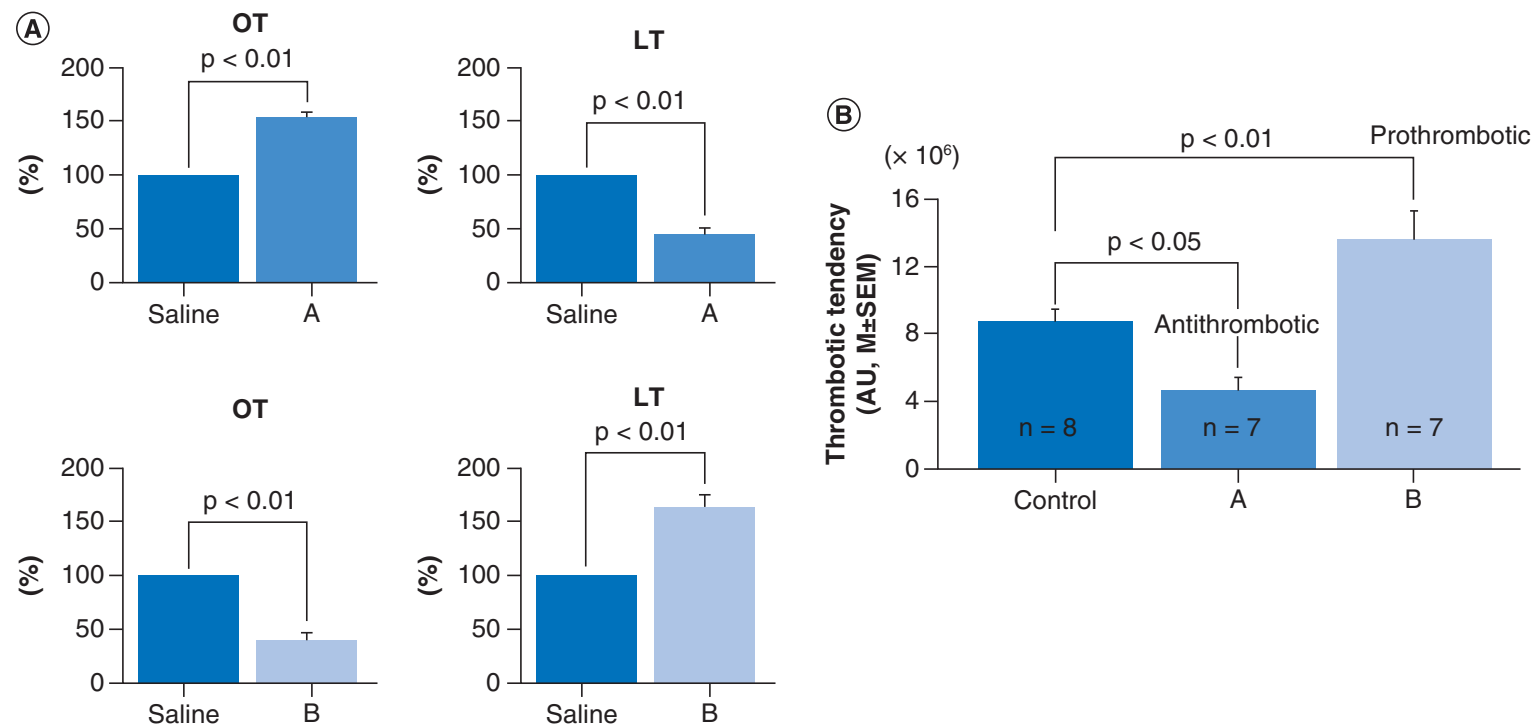

Figure 1. Effect of two grape varieties on thrombosis. (A) Comparison between antithrombotic activity of Cabernet sauvignon (Man) (A) and Neo muscat (B), assessed by the in vitro test (GTT). Mean values with standard error of the mean (SEM) are displayed. P-values are shown relative to saline (control). (B) Comparison of antithrombotic activity of Cabernet sauvignon (Man) (A) and Neo muscat (B), assessed by the in vivo test (laser-induced thrombosis test). Mean values with SEM are displayed. P-values are shown relative to saline (control).

SEM: Standard error of the mean.

\section{Results}

Thrombotic \& thrombolytic activities of red \& white grape varieties assessed by the GTT

These activities and the predicted overall activities are shown in Table 1. OT was 304.5 $\pm 59.3 \mathrm{sec}(\mathrm{Mean} \pm \mathrm{SD})$ and LT $615.7 \pm 84.1 \mathrm{sec}($ Mean $\pm \mathrm{SD})$. As overall thrombotic status is determined by the balance between thrombotic (OT) and thrombolytic (LT) activities, predicted overall activities are shown in Table 1. Correlations between in vitro (GTT) and in vivo (laser-induced test) are shown in Figure 1. All varieties were classified in Table 3 according to the set criteria (Table 2). Measured with the GTT test, Cabernet sauvignon (Man) significantly inhibited thrombotic activity of blood (OT) and enhanced thrombolysis (LT), which suggest that this variety has significant antithrombotic effect. Neo muscat enhanced thrombotic activity of blood (OT) but inhibited thrombolysis (LT) suggesting an overall prothrombotic effect. These effects were confirmed by in vivo thrombosis test (Figure 1).

Cabernet sauvignon (Man) was classified as an antithrombotic grape. Concord, Muscat bailey A (Man) and Honey venus did not show such effect. Most of the red and white grape varieties were prothrombotic.

\section{Effect of heat treatment on thrombotic \& thrombolytic activities}

Heat stability of eight varieties was assessed after their treatment at $100^{\circ} \mathrm{C}$ for $10 \mathrm{~min}$. The treatment changed prothrombotic activity to antithrombotic only in Cabernet franc but in other varieties heat treatment did not affect their thrombotic activities. Results are shown in Table 4.

\section{Polyphenol content in red \& white grape varieties}

Results are shown in Figure 2. Polyphenol contents were very diverse both in red and white grape varieties and the content in red grape varieties was significantly lower than that in white grape varieties.

\section{Correlation between polyphenol content/antioxidant activities \& thrombotic/thrombolytic activities}

Polyphenol content of red and white grape varieties is shown in Figure 2. The contents were diverse in red and white varieties (Figure 2). The content in white grape varieties was significantly higher than that in red grape varieties. Correlation between polyphenol content and thrombotic/thrombolytic activities obtained by the in vitro test (GTT) was analyzed (Figure 3). No significant correlation was shown between OT and polyphenol content. In contrast, significant correlation was shown between LT and polyphenol content. This suggests that polyphenol 
Are all wines made from various grape varieties beneficial in the prevention of myocardial infraction \& stroke? Research Article

Table 1. Effect of grape varieties on thrombosis.

(A) Effect of red grape varieties on thrombotic and thrombolytic activities

Grape variety

Asama Merlot

Dilution

$\mathrm{x} 1$

$x 3$

$\mathrm{x} 10$

Aki queen

$\mathrm{x} 1$

$x 3$

$\mathrm{x} 10$

Oriental star

$x 1$

$x 3$

$\mathrm{x} 10$

Kaiji

$\mathrm{x} 1$

$x 3$

$x 10$

Gamay noir

$\mathrm{x} 1$

$x 3$

Cabernet sauvignon (Man)

$x 10$

$\mathrm{x} 1$

$\mathrm{x} 3$

Cabernet sauvignon (Mer)

$x 10$

$\mathrm{x} 1$

$x 3$

$\mathrm{x} 10$

Cabernet franc

$\mathrm{x} 1$

$x 3$

$x 10$

Campbell early

$\mathrm{x} 1$

$x 3$

$\mathrm{x} 10$

Kyoho

$\mathrm{x} 1$

$x 3$

$\mathrm{x} 10$

Koshu

$\mathrm{x} 1$

$x 3$

$\mathrm{x} 10$

Concord

$\mathrm{x} 1$

$\mathrm{x} 3$

$x 10$

Sunny rouge

$x$

Syrah

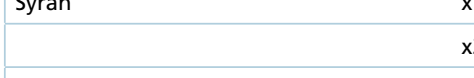

Zenkoji

$x 3$

Mean values ( \pm SEM) are expressed relative to saline (control).

$\dagger^{\dagger} p<0.05$.

$\ddagger \mathrm{p}<0.01$.

ND: Not determined.

\section{Occlusion time (OT)}

$12 \pm 6.9^{\ddagger}$

ND

$91 \pm 2.4$

$22 \pm 8.3^{t}$

ND

$90 \pm 2.6$

$29 \pm 5.7^{\ddagger}$

$52 \pm 9.1^{\ddagger}$

$88 \pm 0.7$

$81 \pm 14.6$

$75 \pm 7.3$

$88 \pm 1.4$

$58 \pm 18.7^{\dagger}$

$35 \pm 17.9^{\dagger}$

$98 \pm 3.0$

$151 \pm 15.2^{\ddagger}$

ND

$97 \pm 5.2$

$62 \pm 9.8^{\dagger}$

$57 \pm 17.0^{\dagger}$

$91 \pm 6.3$

$35 \pm 8.3^{\ddagger}$

$84 \pm 0.6$

$118 \pm 27.8$

$26 \pm 3.6^{\ddagger}$

$39 \pm 206^{\ddagger}$

$68 \pm 17.0$

$27 \pm 7.9 \ddagger$

ND

$86 \pm 2.4$

$69 \pm 6.5^{\ddagger}$

$90 \pm 3.2$

$95 \pm 2.5$

$83 \pm 6.4^{\ddagger}$

$95 \pm 3.6$

$90 \pm 2.7$

$48 \pm 13.5^{\ddagger}$

ND

$83 \pm 1.0$

$40 \pm 6.3^{\ddagger}$

$15 \pm 2.4^{\ddagger}$

$95 \pm 3.2$

$55 \pm 11.0^{\ddagger}$

ND

$91 \pm 3.5$

83

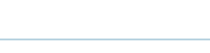

ND

$124 \pm 24.5$

ND

$86 \pm 13.8$

$391 \pm 144.9$

$147 \pm 31.8$

$84 \pm 7.4$

$538 \pm 163.9$

$139 \pm 33.7$

$105 \pm 25.4$

$110 \pm 22.5$

$88 \pm 15.8$

$77 \pm 3.6$

$40 \pm 6.0^{\ddagger}$

ND

$81 \pm 14.3$

$358 \pm 112.6$

$118 \pm 16.9$

$81 \pm 9.6$

$60 \pm 12.0$

$65 \pm 14.3$

$73 \pm 9.1$

$77 \pm 6.6$

$65 \pm 608$

$76 \pm 12.2$

$119 \pm 35.4$

ND

$78 \pm 18.5$

$418 \pm 79.8^{\ddagger}$

$222 \pm 77.6$

$83 \pm 7.9$

$45 \pm 10.3^{\ddagger}$

$59 \pm 3.6^{\dagger}$

$84 \pm 10.0$

$94 \pm 17.7$

ND

$105 \pm 12.3$

$114 \pm 23.8$

$86 \pm 6.7$

$151 \pm 16.1$

$109 \pm 18.2$

ND

$90 \pm 5.0$
Predicted overall activity

Prothrombotic

Prothrombotic

Prothrombotic

Prothrombotic

Prothrombotic

Antithrombotic

Prothrombotic

Prothrombotic

Prothrombotic

Prothrombotic

Prothrombotic

Prothrombotic

not determined

a

Prothrombotic

Prothrombotic

Prothrombotic

Prothrombotic

Prothrombotic


Research Article Iwasaki, Murakami, Ijiri, Shimizu \& Yamamoto

Table 1. Effect of grape varieties on thrombosis (cont.).

(A) Effect of red grape varieties on thrombotic and thrombolytic activities

$\begin{array}{lll}\text { Grape variety } & \text { Dilution } & \text { Occlusion time (OT) }\end{array}$

Charbono $\quad x$

\begin{tabular}{|l|}
\hline $\mathrm{x} 1$ \\
\hline $\mathrm{x} 3$ \\
\hline
\end{tabular}

$100 \pm 6.8$

Lysis time (LT)

Predicted overall activity

$89 \pm 4.7$

$650 \pm 130.4$

Prothrombotic

Delaware

$86 \pm 1.7$

$139 \pm 7.0$

$\mathrm{x} 1$

$118 \pm 6.6$

$x$

ND

ND

Prothrombotic

$\times 3 \quad$ ND

$649 \pm 47.7$

North red

$39 \pm 1.6$

$76 \pm 26.0$

$\mathrm{x} 1 \quad 17 \pm 2.1^{\ddagger}$

$94 \pm 29.6$

$105 \pm 7.6$

\begin{tabular}{|l}
$x 3$ \\
\hline$x 10$
\end{tabular}

$14 \pm 2.1$

$90 \pm 12.9$

Honey seedless

$\mathrm{x} 1$

$73 \pm 14.6$

ND

ND

ND

ND

$117 \pm 14.4$

Honey black

$21 \pm 5.8^{\ddagger}$

$137 \pm 9.1^{\dagger}$

$27 \pm 6.2^{\ddagger}$

ND

ND

$88 \pm 3.4$

$97 \pm 4.0$

$23 \pm 5.2 \ddagger$

$192 \pm 47.1 \ddagger$

Pione $\quad x$

ND

ND

Pinot noir $\quad x 1$

$87 \pm 2.9$

$x 1123 \pm 1.2^{\ddagger}$

$23 \pm 0.7 \ddagger$

$82 \pm 1.3^{\dagger}$

Muscat bailey A (Man)

$x 10$

$24 \pm 9.3^{\ddagger}$

ND

$83 \pm 9.0$

$127 \pm 12.8$

$92 \pm 11.7$

$83 \pm 7.9$

$61 \pm 6.8^{\ddagger}$

Prothrombotic

$x$

$x 3$
$x 10$

$96+6.3$

Muscat bailey A (Mer)

$\mathrm{x} 10$

$96 \pm 6.3$

$35 \pm 7.9 \ddagger$

$88 \pm 1.2$

Muscat bailey A (Nat)

$49 \pm 13.4^{\ddagger}$

ND

$83 \pm 11.2^{\ddagger}$

$29 \pm 5.8^{\ddagger}$

$88 \pm 20.4$

$101 \pm 12.9$

ND

ND

$91 \pm 7.6$

$171 \pm 38.5$

$105 \pm 12.4$

$107 \pm 12.8$

$255 \pm 81.5$

ND

$96 \pm 6.5$

$87 \pm 13.6$

$98 \pm 13.3$

$\mathrm{x} 10$

$109 \pm 17.0$

(B) Effect of white grape varieties on thrombotic and thrombolytic activities

\begin{tabular}{|c|c|c|c|c|}
\hline Grape variety & Dilution & OT & LT & Predicted overall activity \\
\hline \multirow[t]{3}{*}{ Italia } & $\mathrm{x} 1$ & $52 \pm 12.9^{\dagger}$ & $255 \pm 81.5^{\ddagger}$ & Prothrombotic \\
\hline & $x 3$ & $72 \pm 2.7$ & $128 \pm 8.6$ & \\
\hline & $\mathrm{x} 10$ & $89 \pm 1.5$ & $96 \pm 6.5$ & \\
\hline \multirow[t]{3}{*}{ Onsolo } & $\mathrm{x} 1$ & $118 \pm 13.8$ & $140 \pm 47.7$ & No effect \\
\hline & x3 & $84 \pm 1.2$ & $169 \pm 575.2$ & \\
\hline & $\mathrm{x} 10$ & $97 \pm 6.9$ & $273 \pm 100.3$ & \\
\hline \multirow[t]{3}{*}{ Kerner } & $\mathrm{x} 1$ & $21 \pm 3.7^{\ddagger}$ & $84 \pm 17.4$ & Prothrombotic \\
\hline & $x 3$ & $37 \pm 15.5^{\ddagger}$ & $112 \pm 12.6$ & \\
\hline & $x 10$ & $94 \pm 2.4$ & $101 \pm 12.5$ & \\
\hline \multirow[t]{3}{*}{ Shinano riesling } & $\mathrm{x} 1$ & $37 \pm 8.7^{\ddagger}$ & $77 \pm 7.3$ & Prothrombotic \\
\hline & $x 3$ & $89 \pm 1.1$ & $86 \pm 18.1$ & \\
\hline & $\mathrm{x} 10$ & $96 \pm 1.5$ & $116 \pm 38.1$ & \\
\hline \multicolumn{5}{|c|}{$\begin{array}{l}\text { Mean values ( } \pm \text { SEM) are expressed relative to saline (control). } \\
\dagger p<0.05 \text {. } \\
\ddagger p<0.01 . \\
\text { ND: Not determined. }\end{array}$} \\
\hline
\end{tabular}


Are all wines made from various grape varieties beneficial in the prevention of myocardial infraction \& stroke? Research Article

Table 1. Effect of grape varieties on thrombosis (cont.).

(B) Effect of white grape varieties on thrombotic and thrombolytic activities

Grape variety Dilution OT

$x$
$x$

Chardonnay (Man) $\quad x$

$75 \pm 3.3$

$15 \pm 2.1^{\ddagger} \quad 128 \pm 31.7$

$119 \pm 12.0$

$\mathrm{x} 1 \quad 38 \pm 5.1$

$122 \pm 25.9$

$x$

Chardonnay (Mer)

$\times 10$

ND

$162 \pm 13.6^{\ddagger}$

Predicted overall activity

Chardonnay (Mer)

$x 1$
$x 3$

$79 \pm 4.8$

ND

\begin{tabular}{|c|}
\hline Sylvaner \\
\hline
\end{tabular}

$x 10$

$33 \pm 6.4^{\ddagger}$

$111 \pm 9.7$

$33 \pm 6.4^{\ddagger}$
$14 \pm 2.8^{\ddagger}$

$441 \pm 176.5$

Prothrombotic

$87 \pm 2.4$

$158 \pm 11.8$

$145 \pm 70.9$

$34 \pm 6.9 \ddagger$

$132 \pm 23.4$

Prothrombotic

$x 344 \pm 17.0^{\ddagger}$

$102 \pm 9.4$

Sémillon $\quad x$

$98 \pm 4.1$

$94 \pm 14.0$

$x 1 \quad 36 \pm 8.6^{\ddagger}$

$123 \pm 21.6$

Prothrombotic

\begin{tabular}{|c|c|}
\hline$x 3$ \\
\hline$x$
\end{tabular}

Sauvignon blanc $\quad \mathrm{x} 1$

$\mathrm{x} 10$

ND

ND

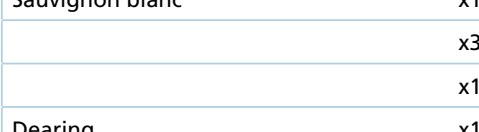

10

$92 \pm 4.7$

$20 \pm 0.2^{\ddagger}$

$88 \pm 14.7$

$\times 3 \quad$ ND

$183 \pm 25.5$

Prothrombotic

$\mathrm{x} 10 \quad 8 \pm 3.2^{\ddagger}$

\begin{tabular}{|l} 
Dearing \\
\hline$x$ \\
$x$
\end{tabular}

Neo muscat

\begin{tabular}{ll} 
Honey venus & $\mathrm{x}$ \\
\hline $\mathrm{x}$ & $\mathrm{x}$ \\
\hline
\end{tabular}

Pannonia kinsce

\begin{tabular}{|l|l}
\hline Pinot blanc & $x$ \\
\hline$x$ \\
\hline
\end{tabular}

Muscat of alexandria

Megumi

\begin{tabular}{|l|c|}
\hline Riesling & $\mathrm{x}$ \\
\hline & $\mathrm{x}$ \\
\hline Müller thurgau & $\mathrm{x}$ \\
\hline & $\mathrm{x}$ \\
\hline $\mathrm{x}$ \\
$\mathrm{x} 3$ \\
$\mathrm{x}$
\end{tabular}

Mean values ( \pm SEM) are expressed relative to saline (control)

$\dagger_{p}<0.05$

$\ddagger p<0.01$.

ND: Not determined.

$\mathrm{x} 1 \quad 21 \pm 3.5^{+}$

$\times 3 \quad 35 \pm 9.7^{\ddagger}$

$\times 10 \quad 103 \pm 4.0$

$\mathrm{x} 1 \quad 57 \pm 5.7^{\ddagger}$

$x 3 \quad 53 \pm 8.8^{\ddagger}$

$\times 10 \quad 86 \pm 3.3$

$x 1 \quad 23 \pm 4.5^{\ddagger}$

$\times 3 \quad$ ND

$\mathrm{x} 10 \quad 85 \pm 2.6$

$\mathrm{x} 1 \quad 28 \pm 7.1^{\ddagger}$

$x 3 \quad 50 \pm 13.4^{\ddagger}$

$\times 10 \quad 89 \pm 3.9$

$\mathrm{x} 1 \quad 48 \pm 5.77^{\ddagger}$

$\times 3 \quad$ ND

$\times 10 \quad 77 \pm 6.8^{\ddagger}$

$\mathrm{x} 1 \quad 21 \pm 3.5^{\ddagger}$

$x 3 \quad 12 \pm 1.0^{\ddagger}$

$\times 10 \quad 87 \pm 2.9$

$\mathrm{x} 1 \quad 28 \pm 4.3^{\ddagger}$

$x 3$ ND

$\times 10 \quad 89 \pm 4.1$

\section{$89 \pm 4.1$}

$62 \pm 16.2^{\ddagger}$

$88 \pm 2.9$

$98 \pm 2.4$

$22 \pm 5.5^{\ddagger}$

ND

$75 \pm 9.1$

ND

$100 \pm 18.3$

$493 \pm 173.0$

$609 \pm 125.6^{\ddagger}$

$169 \pm 65.5$

$679 \pm 59.2^{\ddagger}$

$144 \pm 13.8^{\dagger}$

$124 \pm 6.8$

$77 \pm 11.8^{\dagger} \quad$ Not determined

ND

$79 \pm 4.6$

$150 \pm 27.2^{\dagger} \quad$ Prothrombotic

$113 \pm 20.8$

$90 \pm 5.2$

$68 \pm 16.5 \quad$ Prothrombotic

$$
\text { ND }
$$

$96 \pm 7.6$

$417 \pm 102.7^{\ddagger} \quad$ Prothrombotic

$143 \pm 9.0$

$94 \pm 4.3$

$127 \pm 19.0 \quad$ Prothrombotic

ND

$98 \pm 6.0$

$95 \pm 19.5 \quad$ Prothrombotic

$86 \pm 17.6$

$104 \pm 8.4$

$91 \pm 11.5 \quad$ Prothrombotic

ND

$99 \pm 9.9$ 
Table 2. Criteria of classifying grape varieties

\begin{tabular}{|c|c|c|c|c|}
\hline Dilution factor & Occlusion time (OT) & Index & Occlusion time (OT) & Index \\
\hline $\mathrm{x} 1$ & Not measured by blood running out & +1 & Not measured by blood coagulation & -1 \\
\hline $\mathrm{x} 1$ & Significant increase & +1 & Significant decrease & -1 \\
\hline$x 3$ & Significant increase & +2 & Significant decrease & -2 \\
\hline $\mathrm{x} 10$ & Significant increase & +3 & Significant decrease & -3 \\
\hline$>\times 10$ & Significant increase & +4 & Significant decrease & -4 \\
\hline \multicolumn{5}{|c|}{ (B) Criteria of classifying grape varieties based on thrombolytic activity (LT) } \\
\hline Dilution factor & Lysis time (LT) & Index & Lysis time (LT) & Index \\
\hline $\mathrm{x} 1$ & Not measured by blood running out & +1 & Not measured by blood coagulaton & -1 \\
\hline $\mathrm{x} 1$ & Significant decrease & +1 & Significant increase & -1 \\
\hline x3 & Significant decrease & +2 & Significant increase & -2 \\
\hline $\mathrm{x} 10$ & Significant decrease & +3 & Significant increase & -3 \\
\hline$>\times 10$ & Significant decrease & +4 & Significant increase & -4 \\
\hline
\end{tabular}
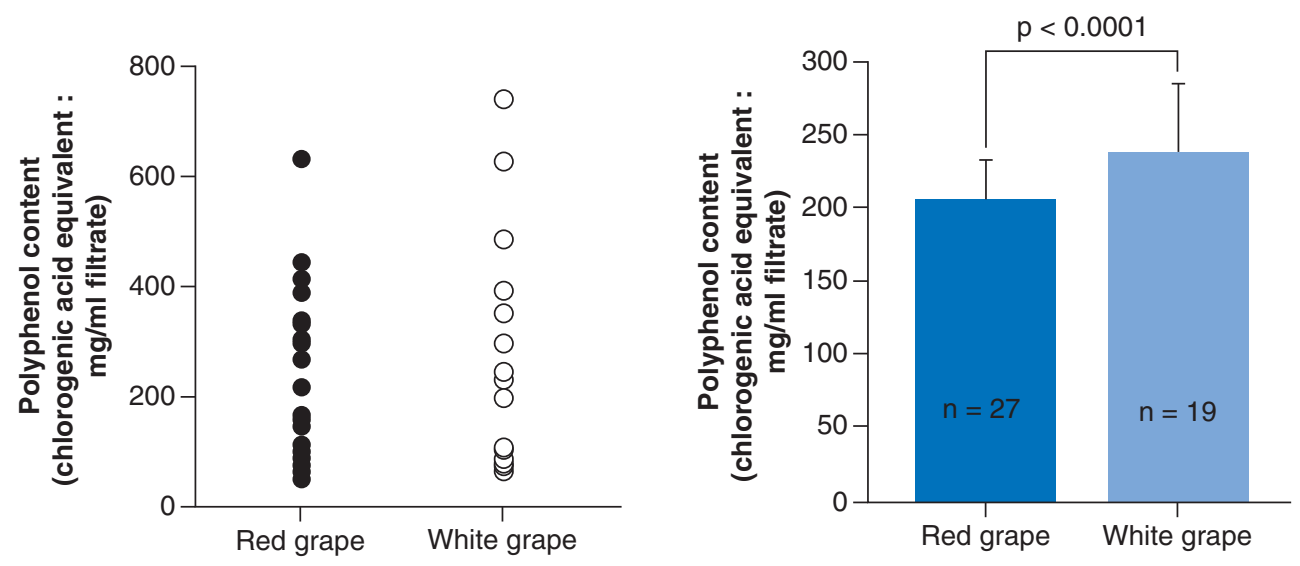

Figure 2. Polyphenol content in red and white grape varieties. Mean values with SEM (error bars) are displayed.
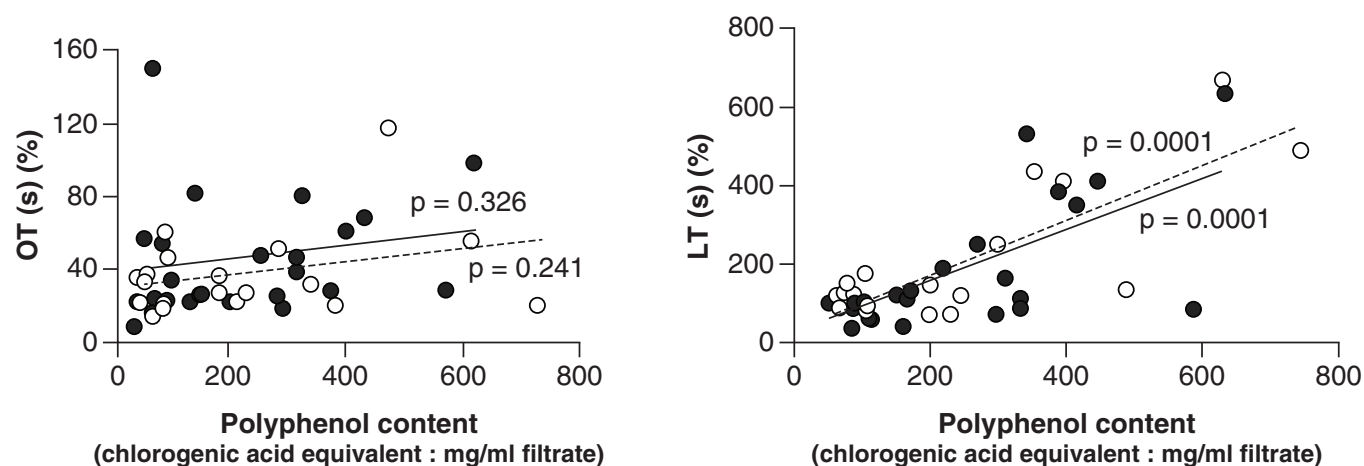

Figure 3. Correlation between polyphenol content and thrombotic (OT)/thrombolytic (LT) activities. P-values in OT in red and white varieties are 0.326 and 0.241 , respectively; p-Values in LT in red and white varieties are 0.0001 and 0.0001 , respectively.

LT: Lysis time; OT: Occlusion time.

enhances overall thrombotic activity both in red and white grape varieties. No significant correlation was found between antioxidant activity and thrombotic/thrombolytic activities. 
Table 3. Antithrombotic activity ranking of grape varieties.

\begin{tabular}{|c|c|}
\hline Intensity & Varieties \\
\hline \multicolumn{2}{|l|}{+2} \\
\hline+1 & Cabernet sauvignon (Man) \\
\hline 0 & Kaiji, Charbono \\
\hline-1 & $\begin{array}{l}\text { Asama merlot, Cabernet franc, Zenkoji, Muscat bailey A (Man), Merlot, Aki queen, Kyoho, Concord, Sunny rouge, Delaware, Honey seedless, Honey } \\
\text { black, Pione, Koshu }\end{array}$ \\
\hline-2 & Gamay noir, Syrah, Oriental star, Campbell early, North red, Cabernet sauvignon (Mer), Muscat bailey A (Mer) \\
\hline-3 & PiNot noir, Muscat bailey A (Nat) \\
\hline \multicolumn{2}{|l|}{ ND } \\
\hline \multicolumn{2}{|c|}{ (B) Antithrombotic activity ranking of white grape varieties based on thrombotic activity (OT) } \\
\hline Intensity & Varieties \\
\hline \multicolumn{2}{|l|}{+1} \\
\hline 0 & Onsolo \\
\hline-1 & Shinano riesling, Chardonnay (Man), Sémillon, Riesling, Müller thurgau, Italia, Honey venus, Pannonia kincse, Megumi \\
\hline-2 & Kerner, Sylvaner, Shine muscat, Dearing, Neo muscat, Muscat of Alexandria, Chardonnay (Mer) \\
\hline-3 & Pinot blanc, Sauvignon branc \\
\hline \multicolumn{2}{|l|}{-4} \\
\hline \multicolumn{2}{|c|}{ (C) Antithrombotic activity ranking of red grape varieties based on thrombolytic activity (LT) } \\
\hline Intensity & Varieties \\
\hline+2 & Concord \\
\hline+1 & Cabernet sauvignon (Man), Muscat bailey A (Man) \\
\hline 0 & Gamay noir, Cabernet franc, Syrah, Zenkoji, Pinot noir, Merlot, Oriental star, Campbell early, Kyoho, Sunny rouge, North red, Aki queen \\
\hline-1 & Kaiji, Charbono, Delaware, Honey seedless, Honey black, Pione, Cabernet sauvignon (Mer), Koshu, Muscat bailey A (Mer) \\
\hline \multicolumn{2}{|l|}{-2} \\
\hline ND & Asama merlot, Muscat bailey A (Nat) \\
\hline \multicolumn{2}{|c|}{ (D) Antithrombotic activity ranking of white grape varieties based on thrombolytic activity (LT) } \\
\hline Intensity & Varieties \\
\hline \multicolumn{2}{|l|}{+2} \\
\hline+1 & Honey venus \\
\hline 0 & Kerner, Shinano riesling, Sylvaner, Sémillon, Sauvignon branc, Megumi, Pinot blanc, Müller thurgau, Riesling, Onsolo, Shine muscat \\
\hline-1 & Chardonnay (Man), Chardonnay (Mer), Italia, Pannonia kincse, Muscat of Alexandria \\
\hline-2 & Neo muscat \\
\hline \multicolumn{2}{|l|}{-3} \\
\hline ND & Dearing \\
\hline
\end{tabular}

LT: Lysis time; OT: Occlusion time.

\section{Discussion}

Since the proposed French paradox hypothesis and findings of various epidemiologic studies, the prevailing idea was that consumption of any red wine is beneficial in the prevention of cardiovascular diseases. Experimental findings using animal models of thrombosis supported such claim [42-45]. These experiments showed that intravenously and orally administered red wine and purple grape juice prepared from some special grape variety inhibited thrombosis in vivo. Similar findings were reported when anticoagulated blood samples were tested in vitro by agonist-induced platelet aggregation tests.

Pathologically relevant point-of-care thrombosis tests were not commercially available for some time. Platelet function (thrombosis) tests commonly in use are testing anticoagulated blood. Flow and shear forces, main determinants of thrombus formation in vivo were not present in these tests. Currently much attention has been focused on a point-of-care shear-induced thrombosis/thrombolysis in vitro test (GTT), which is using for the first time nonanticoagulated blood.

A total of 26 red grape and 19 white grape varieties were tested for antithrombotic effect by the GTT test. This technique can measure not only thrombotic and thrombolytic/fibrinolytic activities but also predict the overall antithrombotic activity [46-48]. This was also confirmed in the present study (Figure 1). Tested by this technique, one 
Table 4. Effect of heating on thrombotic and thrombolytic activities.

\begin{tabular}{|c|c|c|c|c|}
\hline Grape variety & Heat treatment & Occlusion time (OT) & Lysis time (LT) & Predicted overall activity \\
\hline \multirow[t]{2}{*}{ Cabernet sauvignon (Man) } & Before & $127 \pm 14.3^{\dagger}$ & $74 \pm 15.7^{\dagger}$ & Antithrombotic \\
\hline & After & $88 \pm 2.5$ & $34 \pm 4.7^{\ddagger}$ & Less antithrombotic \\
\hline \multirow[t]{2}{*}{ Onsolo } & Before & $121 \pm 18.4$ & $201 \pm 89.0$ & No effect \\
\hline & After & $113 \pm 9.4$ & $386 \pm 116.5$ & No effect \\
\hline \multirow[t]{2}{*}{ Cabernet franc } & Before & $44 \pm 20.7^{\dagger}$ & $55 \pm 6.2$ & Prothrombotic \\
\hline & After & $125 \pm 89.8$ & $47 \pm 13.2^{\dagger}$ & antithrombotic \\
\hline \multirow[t]{2}{*}{ Neo muscat } & Before & $55 \pm 10.3^{\ddagger}$ & $505 \pm 56.3^{\ddagger}$ & Prothrombotic \\
\hline & After & $30 \pm 5.3^{\ddagger}$ & $370 \pm 68.8^{\ddagger}$ & Prothrombotic \\
\hline \multirow[t]{2}{*}{ Pinot blanc } & Before & $30 \pm 4.7^{\ddagger}$ & $97 \pm 11.1$ & Prothrombotic \\
\hline & After & $31 \pm 5.1^{\ddagger}$ & $80 \pm 12.8$ & Prothrombotic \\
\hline \multirow[t]{2}{*}{ Koshu } & Before & $61 \pm 6.1^{\dagger}$ & $461 \pm 104.5^{\ddagger}$ & Prothrombotic \\
\hline & After & $49 \pm 4.1^{\dagger}$ & $239 \pm 113.9$ & Prothrombotic \\
\hline \multirow[t]{2}{*}{ Müller thurgau } & Before & $30 \pm 9.7^{\ddagger}$ & $91 \pm 16.5$ & Prothrombotic \\
\hline & After & $54 \pm 21.1^{\ddagger}$ & $99 \pm 17.4$ & Prothrombotic \\
\hline \multirow[t]{2}{*}{ Shine musca } & Before & $10 \pm 1.9^{\ddagger}$ & $57 \pm 10.9$ & Prothrombotic \\
\hline & After & $10 \pm 0.3^{\ddagger}$ & $82 \pm 26.8$ & Prothrombotic \\
\hline \multicolumn{5}{|c|}{$\begin{array}{l}\text { Filtrates were heated for } 10 \mathrm{~min} \text { at } 100^{\circ} \mathrm{C} \text { and thrombotic and thrombolytic activities were measured by GTT. Values are expressed as relative ones to saline. } \\
{ }_{p} p<0.05 \text {. } \\
\ddagger p<0.01 .\end{array}$} \\
\hline
\end{tabular}

red grape variety Cabernet sauvignon (Man) showed overall antithrombotic activity but the overall antithrombotic effect of two varieties, Concord and Muscat bailey A (Man), was not certain. The latter should be investigated in the future by in vivo tests. All other varieties had no inhibitory effect on thrombus formation, rather they were prothrombotic. In white grape varieties, Honey venus might be antithrombotic but this needs to be confirmed by using in vivo tests. Majority of tested white grape varieties were prothrombotic. All varieties were classified as shown in Table 3 according to the criteria shown in Table 2. The inconsistency between Cabernet sauvignon (Man) and Cabernet sauvignon (Mer) might derive from their suppliers or vine trees. This problem remains to be solved in the future.

Heat stability of the experimental antithrombotic effect are shown in Table 4. The effect of most varieties was heat stable but in Cabernet franc the thrombotic activity changed from prothrombotic to antithrombotic by heat treatment.

Folts and colleagues suggested that antithrombotic activity may be due to polyphenol and antioxidant content of wines [42-45]. For this reason, in the present study the relationship between GTT-measured thrombotic (OT)/thrombolytic activities (LT) and polyphenol content/antioxidant activity of grape varieties were also investigated. Polyphenol content had no significant relation to the effect on thrombus formation, but it had an inhibition on thrombolysis.

In this study we selected the chlorogenic acid as representative of polyphenolic acids on the grounds that quite a few published studies did the same. However, it should be considered that chlorogenic acid were not found in grapes but in coffee beans. Our findings, that chlorogenic acid contents significantly relate to the thrombolytic activity of grape extract may stimulate future investigation of using polyphenolic acid representative other the chlorogenic acid to clarify this issue.

The antioxidant activity of grapes had no significant relation to either thrombosis or thrombolysis.

In an earlier study of mulberry, it was found that antioxidants had inhibitory effect on thrombosis [46]. Polyphenols inhibited thrombosis in carrots [47]. Antioxidant activity and polyphenol content had no significant relation to thrombosis (OT) and thrombolysis (LT) in apple [48]. Lycopene had no significant relation to the antithrombotic activity [49]. These inconsistencies together with the present findings do not support the assumption that these components determine the antithrombotic activity of fruits and vegetables. This is however not inconsistent with findings that certain purified components had antithrombotic effects [51-54]. We have to pay attention to the finding that filtrates of different grape varieties are equivalent to the different wines. Simple phenols might be transformed into complex molecules during wine production and wine aging. Moreover, by filtration of grapes only compounds 
present in the pulp are extracted, but not polyphenols which are present in the skin and seeds, which can be extracted only by maceration during wine making. However, the assumption that all red wine is antithrombotic should be put to further investigation.

We have selected fruit and vegetable varieties with experimental antithrombotic effect [55-60] and demonstrated that oral intake of these varieties can inhibit thrombotic status in humans [61,62].

\section{Conclusion}

The aim of the present study was to determine whether only red wines and not white wines have antithrombotic effect. In this study grape juices prepared from various red and white grape varieties were tested by pathologically relevant in vitro and in vivo thrombosis test. The study demonstrated that both red and white grape juices obtained from various varieties can have antithrombotic effect and such effect is dependent on the variety of tested grape. As such, the present findings challenge the French paradox hypothesis and put it into a new light.

\section{Future perspective}

According to the 'French paradox' the popular consumption of red wines, antithrombotic effect is solely responsible for the low incidents of coronary heart disease and stroke in the French population. The present study confirms this assumption but found some white wines also having antithrombotic effect. Thus, wines from special varieties of grapes, together with intake of antithrombotic fruit and vegetable varieties may constitute an antithrombotic diet, effective in the prevention of myocardial infarction or stroke.

\section{Summary points}

- Antithrombotic activity of 26 red grape varieties and nineteen white grape varieties were measured by an in vitro test which use nonanticoagulated blood (Global Thrombosis Test), and also by an animal model of thrombosis.

- In general, the antithrombotic effect or the lack of it depended on the variety of the tested grapes.

- Both red and white wine from specific varieties may have antithrombotic effect, while the majority of wines do not have significant effect on thrombotic activity of blood.

- Our findings put the French paradox hypothesis into new light.

\section{Author contributions}

J Yamamoto designed the study. M Iwasaki performed the experiments as a part of his Master's thesis in Kobe Gakuin University published in 2006. The thesis is written in Japanese and kept in the Kobe Gakuin University Library. None can access it via internet. M Murakami, Y ljiri and M Shimizu reviewed and revised the manuscript.

\section{Acknowledgments}

The authors would like to thank Rob Furlong, Shropshire, UK, and Shinji Yamamoto, Nihon Fukushi University, Aichi, Japan, for revising English and preparing Tables, respectively.

\section{Financial \& competing interests disclosure}

The authors have no relevant affiliations or financial involvement with any organization or entity with a financial interest in or financial conflict with the subject matter or materials discussed in the manuscript. This includes employment, consultancies, honoraria, stock ownership or options, expert testimony, grants or patents received or pending, or royalties.

No writing assistance was utilized in the production of this manuscript.

\section{Ethical conduct of research}

Animals were maintained in compliance with the "Guiding Principles for the Care and Use of Animals in the field of Physiological Sciences", published by Physiological Society of Japan. The protocol was approved by the Animal Experiment Committee of Kobe Gakuin University.

Open access

This work is licensed under the Creative Commons Attribution 4.0 License. To view a copy of this license, visit http://creativecomm ons.org/licenses/by/4.0/ 


\section{References}

1. Violi F, Pastori D, Pignatelli P, Carnevale R. Nutrition, thrombosis, and cardiovascular disease. Circ. Res. 126(10), 1415-1442 (2020).

2. Liu S, Manson JE, Lee IM et al. Fruit and vegetable intake and risk of cardiovascular disease: the women's health study. Am. J. Clin. Nutr. 72(4), 922-928 (2000).

3. Joshipura KJ, Hu FB, Manson JE et al. The effect of fruit and vegetable intake on risk for coronary heart disease. Ann. Intern. Med. 134(12), 1106-1114 (2001).

4. Bazzano LA, He J, Ogden LG et al. Fruit and vegetable intake and risk of cardiovascular disease in US adults: the first national health and nutrition examination survey epidemiologic follow-up study. Am. J. Clin. Nutr. 76(1), 93-99 (2002).

5. He FJ, Nowson CA, MacGregor GA. Fruit and vegetable consumption and stroke: meta-analysis of cohort studies. Lancet 367(9507), 320-326 (2006).

6. Food-based dietary guidelines (Europe). www.euro.who.int/__data/assets/pdf_file/0017/150083/E79832.pdf

7. Key Elements of Healthy Eating Patterns (United States). https://health.gov/dietaryguidelines/2015/guidelines/chapter-1/a-closer-look-inside-healthy-eating-patterns/\#table-1--1

8. Food-based dietary guidelines (Japan). http://www.fao.org/nutrition/education/food-dietary-guidelines/regions/countries/Japan/en

9. Yamamoto J, Ijiri Y, Ikarugi H, Otsui K, Inoue N, Sakariassen KS. Prevention of thrombotic disorders by antithrombotic diet and exercise: evidence by using global thrombosis tests. Future Sci. OA 4(4), FSO285 (2018).

10. Satija A, Bhupathiraju SN, Spiegelman D et al. Healthful and unhealthful plant-based diets and the risk of coronary heart disease in U.S. adults. J. Am. Coll. Cardiol. 70(4), 411-422 (2017).

11. Harrison P. Platelet function analysis. Blood Rev. 19(2), 111-123 (2005).

12. Paniccia R, Priora R, Liotta AA, Abbate R. Platelet function tests: a comparative review. Vasc. Health Risk Manag. 11, 133-148 (2015).

13. Gorog DA. Prognostic value of plasma fibrinolysis activation markers in cardiovascular disease. J. Am. Coll. Cardiol. 55(24), 2701-2709 (2010).

14. Sakariassen KS, Aarts PAMM, de Groot PG, Houdijk WPM, Sixma JJ. A perfusion chamber developed to investigate platelet interaction in flowing blood with human vessel wall cells, their extracellular matrix and purified components. J. Lab. Clin. Med. 102(4), 522-535 (1983).

15. Barstad RM, Roald HE, Cui W, Turitto VT, Sakariassen KS. A perfusion chamber developed to investigate thrombus formation and shear profiles in flowing native blood at the apex of well-defined stenoses. Arterioscler. Thromb. 14(12), 1984-1991 (1994).

16. Sakariassen KS, Turitto VT, Baumgartner HR. Recollections of the development of flow devices for studying mechanisms of hemostasis and thrombosis in flowing whole blood. J. Thromb. Haemost. 2(10), 1681-1690 (2004).

17. Sakariassen KS. Thrombus formation on apex of arterial stenoses: the need for a fluid high shear diagnosis device. Future Cardiol. 3(2), 193-201 (2007).

18. Sakariassen KS, Orning L, Turitto VT. The impact of blood shear rate on arterial thrombus formation. Future Sci. OA 1(4), FSO30 (2015).

19. Gorog DA, Kovacs IB. Modeling coronary thrombosis from non-anticoagulated human blood in vitro. Hematol Pathol 4, 43-52 (1990).

20. Ratnatunga CP, Edmondson SF, Rees GM, Kovacs IB. High-dose aspirin inhibits shear-induced platelet reaction involving thrombin generation. Circulation 85(3), 1077-1082 (1992).

21. Gorog DA, Kovacs IB. Thrombotic status analyser. Measurement of platelet-rich thrombus formation and lysis in native blood. Thromb Haemost. 73(3), 514-520 (1995).

22. Ostrowska M, Gorog DA. Does morphine remain a standard of care in acute myocardial infarction? Med. Res. J. 5(1), 46-49 (2020).

23. Gue YX, Inoue N, Spinthakis N et al. Thrombotic profile and oral anticoagulation in asian and non-asian patients with nonvalvular atrial fibrillation. J. Am. Coll. Cardiol. 74(22), 2822-2824 (2019).

24. Gue YX, Spinthakis N, Farag M et al. Impact of preadmission morphine on reinfarction in patients with ST-elevation myocardial infarction treated with percutaneous coronary intervention: a meta-analysis. Clin. Pharmacol. Ther. (2020). doi:10.1002/cpt.1798 (Epub ahead of print).

25. Gorog DA, Farag M, Spinthakis N et al. Effect of remote ischaemic conditioning on platelet reactivity and endogenous fibrinolysis in ST-elevation myocardial infarction: a substudy of the CONDI-2/ERIC-PPCI randomized controlled trial. Cardiovasc. Res. (2020) doi:10.1093/cvr/cvaa061 (Epub ahead of print).

26. Ahn JH, Gorog DA, Jeong YH, Geisler T. Racial differences in level of inflammation and clinical outcomes between European and east Asian patients undergoing percutaneous coronary intervention: the compare-CRP study. J. Am. Coll. Cardiol. 75 (11), 24 (2020).

27. Gorog DA, Geisler T. Platelet inhibition in acute coronary syndrome and percutaneous coronary intervention: insights from the past and present. Thromb. Haemost. 120(4), 565-578 (2020).

28. Liberale L, Gorog DA. Low-grade endotoxaemia and platelets: a deadly aggregation. Eur. Heart J. (2020). doi.org/10.1093/eurheartj/ehz955 (Epub ahead of print). 
29. Otsui K, Gorog DA, Yamamoto J et al. Global thrombosis test - a possible monitoring system for the effects and safety of dabigatran. Thromb. J. 13, 39 (2015). doi:10.1186/s12959-015-0069-6

30. Okafor ON, Gorog DA. Endogenous fibrinolysis: an important mediator of thrombus formation and cardiovascular risk. J. Am. Coll. Cardiol. 65(16), 1683-1699 (2015).

31. Gorog DA, Otsui K, Inoue N. Usefulness of platelet function tests to predict bleeding with antithrombotic medications. Cardiol. Rev. 23(6), 323-327 (2015).

32. Yamamoto J, Inoue N, Otsui K, Ishii H, Gorog DA. Global thrombosis test (GTT) can detect major determinants of haemostasis including platelet reactivity, endogenous fibrinolytic and thrombin generating potential. Thromb. Res. 133(5), 919-926 (2014).

33. Suehiro A, Wakabayashi I, Yamashita T, Yamamoto J. Attenuation of spontaneous thrombolytic activity measured by the global thrombosis test in male habitual smokers. J. Thromb. Thrombolysis 37(4), 414-418 (2014).

34. Suehiro A, Wakabayashi I, Uchida K, Yamashita T, Yamamoto J. Impaired spontaneous thrombolytic activity measured by global thrombosis test in males with metabolic syndrome. Thromb. Res. 129(4), 499-501 (2012).

35. Gorog DA, Yamamoto J, Saraf S et al. First direct comparison of platelet reactivity and thrombolytic status between Japanese and Western volunteers: possible relationship to the "Japanese paradox". Int. J. Cardiol. 152(1), 43-48 (2011).

36. Taomoto K, Ohnishi H, Kuga Y et al. Platelet function and spontaneous thrombolytic activity of patients with cerebral infarction assessed by the global thrombosis test. Pathophysiol. Haemost. Thromb. 37(1), 43-48 (2010).

37. Yamashita T, Sato A, Ikarugi H, Inoue A, Kitamori K, Ishii H, Yamamoto J. Significantly reduced spontaneous thrombolytic activity in older men: a possible explanation for the gender differences in risk of acute coronary syndromes. Thromb. Res. 116(2), 127-131 (2005).

38. Ikarugi H, Shibata M, Shibata S, Ishii H, Taka T, Yamamoto J. High intensity exercise enhances platelet reactivity to shear stress and coagulation during and after exercise. Pathophysiol. Haemost. Thromb. 33(3), 127-133 (2003).

39. Ikarugi H, Yamashita T, Aoki R, Ishii H, Kanki K, Yamamoto J. Impaired spontaneous thrombolytic activity in elderly and in habitual smokers, as measured by a new global thrombosis test. Blood. Coagul. Fibrinolysis 14(8), 781-784 (2003).

40. Yamamoto J, Yamashita T, lkarugi $\mathrm{H}$ et al. Gorog thrombosis test: a global in vitro test of platelet function and thrombolysis. Blood. Coagul. Fibrinolysis 14(1), 31-39 (2003).

41. Renaud S, De Lorgeril M. Wine, alcohol, platelets, and the French paradox for coronary heart disease. Lancet 339(8808), $1523-1526$ (1992).

42. Demrow HS, Slane PR, Folts JD. Administration of wine and grape juice inhibits in vivo platelet activity and thrombosis in stenosed canine coronary arteries. Circulation 91(4), 1182-1188 (1995).

43. Osman HE, Maalej N, Shanmuganayagam D, Folts JD. Grape juice but not orange or grapefruit juice inhibits platelet activity in dogs and monkeys. J. Nutr. 128(12), 2307-2312 (1998).

44. Keevil JG, Osman HE, Reed JD, Folts JD. Grape juice, but not orange juice or grapefruit juice, inhibits human platelet aggregation. $J$. Nutr. 130(1), 53-56 (2000).

45. Freedman JE, Parker C 3rd, Li L et al. Select flavonoids and whole juice from purple grapes inhibit platelet function and enhance nitric oxide release. Circulation 103(23), 2792-2798 (2001).

46. Yamamoto J, Naemura A, Ura M et al. Testing various fruits for anti-thrombotic effect: I. Mulberries. Platelets 17(8), 555-564 (2006).

47. Yamamoto J, Naemura A, Ijiri Y et al. The antithrombotic effects of carrot filtrates in rats and mice. Blood Coagul. Fibrinolysis 19(8), 785-792 (2008).

48. Morishita M, Naemura A, Tamura Y et al. Mechanism of the experimental antithrombotic effect of some apple varieties involves enhanced endogenous thrombolytic activity. Interv. Med. Appl. Sci. 4(3), 115-124 (2012).

49. Yamamoto J, Ohno H, Hyodo K, Onishi M, Giddings J. The antithrombotic activity of mini-type tomatoes is dependent on the particular variety and the stage of harvest. Lycopene content does not contribute to antithrombotic activity. Health 5(4), 681-686 (2013).

50. Ijiri $Y$, Miura M, Hashimoto $M$ et al. A new model to evaluate the diet-induced prothrombotic state, using He-Ne laser-induced thrombogenesis thrombogenesis in the carotid artery of apolipoprotein E-deficient and low-density lipoprotein receptor-deficient mice. Blood Coagul. Fibrinolysis 13(6), 497-504 (2002).

51. Cao AH, Wang J, Gao HQ, Zhang P, Qiu J. Beneficial clinical effects of grape seed proanthocyanidin extract on the progression of carotid atherosclerotic plaques. J. Geriatr. Cardiol. 12(4), 417-423 (2015).

52. Sano T, Oda E, Yamashita T et al. Anti-thrombotic effect of proanthocyanidin, a purified ingredient of grape seed. Thromb. Res. 115(1-2), 115-21 (2005).

53. Fukao H, Ijiri Y, Miura M et al. Effect of trans-resveratrol on the thrombogenicity and atherogenicity in apolipoprotein E-deficient and low-density lipoprotein receptor-deficient mice. Blood Coagul. Fibrinolysis. 15(6), 441-446 (2004).

54. Yamamoto J, Ijiri Y, Tamura Y, Iwasaki M, Murakami M, Okada Y. Reevaluation of antithrombotic fruits and vegetables: great variation between varieties. Drug Discov. Ther. 10(3), 129-140 (2016).

55. Yamamoto J, Masuda M, Hyodo K, Iwasaki M. Experimental antithrombotic effect of potatoes harvested in the autumn. Health 4(2), 108-112 (2012). 
56. Kinugasa C, Naemura A, Hyodo K, Nakai Y, Katsuta M, Yamamoto J. Experimental antithrombotic effects of sesame seed whole grains and extracts. Blood Coagul. Fibrinolysis 22(6), 526-531 (2011).

57. Hyodo K, Horii I, Nishino M, Giddings J, Yamamoto J. The antithrombotic effects of onion filtrates in rats and mice. Health 3(6), 319-325 (2011).

58. Naemura A, Mitani T, Ijiri Y et al. Anti-thrombotic effect of strawberries. Blood Coagul. Fibrinolysis 16(7), 501-509 (2005).

59. Yamada K, Naemura A, Sawashita N, Noguchi Y, Yamamoto J. An onion variety has natural antithrombotic effect as assessed by thrombosis/thrombolysis models in rodents. Thromb. Res. 114(3), 213-220 (2004).

60. Yamamoto J, Taka T, Yamada K et al. Tomatoes have natural anti-thrombotic effects. Br. J. Nutr. 90(6), 1031-1038 (2003).

61. Naemura A, Ohira H, Ikeda M, Koshikawa K, Ishii H, Yamamoto J. An experimentally antithrombotic strawberry variety is also effective in humans. Pathophysiol. Haemost. Thromb. 35(3), 398-404 (2006).

62. Ijiri Y, Ishii H, Yamamoto J. Diet of fruits and vegetables with experimental antithrombotic effect may be beneficial to humans in the prevention of arterial thrombotic diseases. Int. J. Drug Dev. Res. 8(3), 6-10 (2016). 